Preprint typeset in JHEP style. - PAPER VERSION

YITP-SB 01-62

\title{
An analytic torsion for graded D-branes
}

\author{
C. I. Lazaroiu \\ C. N. Yang Institute for Theoretical Physics \\ SUNY at Stony BrookNY11794-3840, U.S.A. \\ calin@insti.physics.sunysb.edu
}

\begin{abstract}
I consider the semiclassical approximation of the graded Chern-Simons field theories describing certain systems of topological A type branes in the large radius limit of Calabi-Yau compactifications. I show that the semiclassical partition function can be expressed in terms of a certain (differential) numerical invariant which is a version of the analytic torsion of Ray and Singer, but associated with flat graded superbundles. I also discuss a 'twisted' version of the Ray-Singer norm, and show its independence of metric data. As illustration, I consider graded D-brane pairs of unit relative grade with a scalar condensate in the boundary condition changing sector. For the particularly simple case when the reference flat connections are trivial, I show that the generalized torsion reduces to a power of the classical Ray-Singer invariant of the base 3-manifold.
\end{abstract}




\section{Contents}

$\begin{array}{ll}\text { 1. Introduction } & 2\end{array}$

2. Graded Chern-Simons theories and topological D-branes 3

2.1 The string field theory of graded topological A-branes 3

2.2 D-brane interpretation of the background 7

3. The semiclassical approximation and generalized Ray-Singer torsion 8

3.1 Hermitian structure 8

3.1.1 The conjugation operator 9

3.1.2 The adjoint of $d$ and the Laplacian 9

3.2 The spacetime ghost grading 9

3.3 Complex and real determinants and induced Euclidean scalar products 10

3.4 The semiclassical partition function in the resolvent formalism 11



3.4.2 Generalized Ray-Singer torsion $\quad 15$

3.4.3 A Hermitian analogue of the Ray-Singer metric 16

3.4.4 Metric induced on the real determinant line 18

3.4.5 Metric-independence of the Ray-Singer norm 20

$\begin{array}{lll}3.4 .6 & \text { The complex prefactor } & 20\end{array}$

4. Example: D-brane pairs of unit relative grade in a scalar background 21

4.1 The case of trivial flat bundles 23 


\section{Introduction}

D-brane composite formation is a subject of central importance for a deeper understanding of open string theory dynamics. Of particular interest is the incarnation of such processes for the case of superstring compactifications on Calabi-Yau backgrounds, which provide a rich source of potential phenomenological applications.

A systematic study of D-brane composites is best performed with the tools of string field theory. Indeed, such processes involve off-shell string dynamics, which is best captured in string field language. Given the complexity superstring models, a satisfactory formulation of superstring field theory is lacking Calabi-Yau backgrounds. However, part of the dynamics of spacetime fields originating from the chiral primary sector allows for an explicit description in terms of topological strings[37, 38, 32].

A fundamental observation made in [22](see also [30] and [33]) is that D-branes in Calabi-Yau backgrounds are graded objects. In our context, this means that topological D-branes will carry certain integral data which specify a branch of their 'BPS grade', which was discussed at length in [22]. It was recently proposed [23, 24, 26, 39, 19] that the dynamics of graded topological D-branes is described by certain target space theories which have the form of 'graded Chern-Simons models' ${ }^{1}$. These are versions of Chern-Simons field theories associated with graded superbundles, and whose fields assemble into a 'graded superconnection of total degree one'. This approach, which is intimately connected with the derived category program of [31] and [22, 25, 27], allows for a description of topological D-brane physics in standard field theoretic terms. It also leads to a physical representation of the extended moduli space of open strings $[19,2]$.

One benefit of this description is that the formal analogy between graded and ungraded Chern-Simons theories suggests a wealth of generalizations of classical connections between physics and certain mathematical invariants. In this note, we take a first step in this direction for the graded Chern-Simons theory of A-type branes.

It is well-known that the semiclassical approximation of usual Chern-Simons theory (which forms the string field theory of single, ungraded topological A-branes) is related to the analytic torsion of Ray and Singer. In fact, the Chern -Simons approach allows one to 'discover' the torsion from physical considerations. It is natural to ask what is the analogue of this relation for graded Chern-Simons models. Proceeding in physical manner, we consider the semiclassical approximation of graded Chern-Simons theories, from which we extract a generalized version of Ray-Singer torsion, which should

\footnotetext{
${ }^{1}$ For the A-model, these theories do not take into account worldsheet instanton corrections (which can be formally incorporated along the lines of $[28,29])$. In the present paper, we always work in the large radius limit of a given Calabi-Yau compactification, where such corrections can be neglected.
} 
prove useful for a topological characterization of D-brane composites in Calabi-Yau backgrounds.

The note is organized as follows. In Section 2, we give a short review of the graded Chern-Simons theory of $[39,19]$ and recall the D-brane interpretation of its vacuum configurations. In Section 3, we consider the semiclassical approximation to these models around a general background. Upon using the methods of $[8,9,12]$ and related work, we evaluate the partition function in this limit up to a real prefactor related to the isotropy subgroup of the background. This allows us to express the result in terms of a certain generalization of the analytic torsion of Ray and Singer $[5,6,7]$. This numerical invariant is related to (but does not seem to coincide with) a quantity considered in [34]. Upon writing the semiclassical partition function in terms of a 'twisted Ray-Singer metric', we use general results of $[9,12]$, in order to show that the extended Ray-Singer norm is independent of the metric data employed in the gauge-fixing procedure. We also discuss the argument of the semiclassical partition function, which displays a metric dependence reminiscent of that known from usual Chern-Simons theory. Section 4 considers the generalized torsion of graded D-brane pairs (of unit relative grade) in the presence of a scalar condensate. For the case of trivial underlying flat connections, we show that the generalized torsion reduces to a power of the classical Ray-Singer invariant of the three-cycle.

Conventions There exist a few different conventions for the Ray-Singer torsion in the literature. In this note, we define the Ray-Singer torsion of a closed three-manifold $L$ (with respect to the trivial flat line bundle) by:

$$
T(L)=\prod_{q} \operatorname{det}^{\prime}, r e g\left(\Delta_{q}\right)^{\frac{q(-1)^{q}}{2}}
$$

where $\Delta_{q}$ is the $q$-form Laplacian and $\operatorname{det}^{\prime}{ }^{\prime}$ reg $\left(\Delta_{q}\right)$ is the regularized determinant of the restriction to the orthocomplement of its kernel. Other papers define the RaySinger torsion to be $T(L)^{-1}, T(L)^{2}$ or $T(L)^{-2}$, where $T(L)$ is the quantity in (1.1). Our conventions for the generalized torsion will be an extension of (1.1).

\section{Graded Chern-Simons theories and topological D-branes}

\subsection{The string field theory of graded topological A-branes}

Consider a special Lagrangian 3-cycle $L$ in a a Calabi-Yau threefold $X$, endowed with the 'fundamental' orientation discussed in [39, 2]. Given a collection of graded topological branes (of different grades $n$ ) wrapping $L$, we form the total bundle $\mathbf{E}=\oplus_{n} E_{n}$, 
endowed with the $\mathbb{Z}$-grading induced by $n$. Here $E_{n}$ are flat bundles which describe the worldvolume backgrounds of the various topological D-branes. Throughout this paper, we shall assume that the system contains a finite number of graded D-branes, i.e. $n$ takes values in a finite set of integers. We also make the convention that a form on $L$ of rank lying outside the interval $0 . .3$ is defined to be zero. Note that we consider complex flat vector bundles $E_{n}$, which are not required to be unitary (i.e. there need not exist metrics on $E_{n}$ which are covariantly-constant with respect to the flat connections).

The graded Chern-Simons theory of $[39,19]$ describes sections $u$ of the bundle:

$$
\mathcal{V}=\Lambda^{*}\left(T^{*} L\right) \otimes \operatorname{End}(\mathbf{E})
$$

which we endow with the total grading $\mathcal{V}=\oplus_{t} \mathcal{V}^{t}$, where:

$$
\mathcal{V}^{t}=\underset{\substack{k, m, n \\ k+n-m=t}}{ } \Lambda^{k}\left(T^{*} L\right) \otimes \operatorname{Hom}\left(E_{m}, E_{n}\right)
$$

The degree of $u$ with respect to this grading is:

$$
|u|=r k u+\Delta(u)
$$

where $\Delta(u)=n-m$ if $u \in \Omega^{*}\left(L, \operatorname{Hom}\left(E_{m}, E_{n}\right)\right)$. The grading |.| is related (after localization) to the worldsheet $U(1)$ charge of topological string states. The space $\mathcal{H}=$ $\Gamma(\mathcal{V})$ of sections of $\mathcal{V}$ is total boundary space of [39], and can be viewed as the collection of open string states of the system. It is endowed with the grading $\mathcal{H}^{k}=\Gamma\left(\mathcal{V}^{k}\right)$.

Triple string interactions are described by the so-called total boundary product, which is defined through:

$$
u \bullet v=(-1)^{\Delta(u) r k v} u \wedge v
$$

where the wedge product on the right hand side includes composition of morphisms in $\operatorname{End}(\mathbf{E})$. This associative product is compatible with the grading and admits the identity endomorphism of $\mathbf{E}$ as a neutral element:

$$
|u \bullet v|=|u|+|v|, \quad 1 \bullet u=u \bullet 1=u
$$

(note that $|1|=0$ ).

The direct sum $A^{(0)}=\oplus_{n} A_{n}$ of the flat connections on $E_{n}$ induces a flat structure on $\operatorname{End}(\mathbf{E})$. If $d^{(0)}$ is de Rham differential twisted by this flat connection, then it acts as a degree one derivation of the boundary product (since the connection induced on $\operatorname{End}(\mathbf{E})$ is in the 'adjoint representation'). To allow for more general backgrounds, we shift by elements $\phi \in \mathcal{H}^{1}$, which allows us to build the object $d=d^{(0)}+[\phi, .]_{\bullet}$, where 
$[.,.] \bullet$ stands for the graded commutator ${ }^{2}$ in the associative algebra $(\mathcal{H}, \bullet)$. It is clear that $d$ is a degree one derivation of $(\mathcal{H}, \bullet)$ (since so are both terms in its definition):

$$
|d u|=|u|+1 \quad, \quad d(u \bullet v)=(d u) \bullet v+(-1)^{|u|} u \bullet(d v) .
$$

In the language of [34], $d$ is a 'graded superconnection of total degree one'. We refer the reader to [35] for basic facts regarding superconnections. For what follows, we shall pick a reference flat $^{3}$ graded superconnection $d$ (of degree one) which need not coincide with the original flat connection $A^{(0)}$; the formalism is independent of this choice.

One also has a graded trace on $\Omega^{*}(L, \operatorname{End}(\mathbf{E}))$, which is defined through:

$$
\operatorname{str}(u)=\sum_{n}(-1)^{n} \operatorname{tr}\left(u_{n n}\right), \text { for } u=\oplus_{m, n} u_{m n},
$$

with $u_{m n} \in \Omega^{*}\left(L, \operatorname{Hom}\left(E_{m}, E_{n}\right)\right)$. The bilinear form:

$$
\langle u, v\rangle:=\int_{L} \operatorname{str}(u \bullet v)
$$

is non-degenerate and has the properties:

$$
\langle u, v\rangle=(-1)^{|u||v|}\langle v, u\rangle, \quad\langle d u, v\rangle+(-1)^{|u|}\langle u, d v\rangle=0 \quad, \quad\langle u \bullet v, w\rangle=\langle u, v \bullet w\rangle
$$

It also obeys the selection rule $\langle u, v\rangle=0$ unless $|u|+|v|=3$, so that the sign prefactor in the first equation of (2.9) is always one (though it is more convenient to write it out explicitly, as we did).

The string field theory of $[39,19]$ is described by the action ${ }^{4}$ :

$$
S(\phi)=\int_{L} \operatorname{str}\left[\frac{1}{2} \phi \bullet d \phi+\frac{1}{3} \phi \bullet \phi \bullet \phi\right]+c c=\frac{1}{2}\langle\phi, d \phi\rangle+\frac{1}{3}\langle\phi, \phi \bullet \phi\rangle+c c \quad,
$$

which is defined on the degree one component

$$
\mathcal{H}^{1}=\{\phi \in \mathcal{H}|| \phi \mid=1\}=\Gamma\left(\oplus_{k+n-m=1} \Lambda^{k}\left(T^{*} L\right) \otimes \operatorname{Hom}\left(E_{m}, E_{n}\right)\right)
$$

of the total boundary space. This defines a 'graded Chern-Simons field theory', which governs the dynamics of background shifts $\phi$. The equations of motion:

$$
d \phi+\frac{1}{2}[\phi, \phi] \bullet=0 \Leftrightarrow d \phi+\phi \bullet \phi=0 \quad\left(\phi \in \mathcal{H}^{1}\right)
$$

\footnotetext{
${ }^{2}$ This is given by $[u, v] \bullet:=u \bullet v-(-1)^{|u||v|} v \bullet u$.

${ }^{3}$ Flatness means that $d^{2}=0$, and will be required by the equations of motion.

${ }^{4}$ This is the real part of the complex action considered in [2]. In that paper, we were interested in the moduli space, which can be understood without worrying about the real and imaginary parts. In the present note, we shall consider the path integral, which requires that we work with the physical action, an object which must always be real.
} 
are equivalent with the requirement that the shifted superconnection $d_{\phi}=d+[\phi,.] \bullet$ satisfies $\left(d_{\phi}\right)^{2}=0$. Hence the extrema of (2.10) describe flat superconnections of total degree one.

The equations of motion (2.12) are invariant ${ }^{5}$ with respect to gauge transformations of the form:

$$
\phi \rightarrow \phi^{g}=g \bullet \phi \bullet g^{-1}+g \bullet d g^{-1}
$$

where $g$ is an invertible element of the subalgebra $\left(\mathcal{H}^{0}, \bullet\right)$. This means that $g$ belongs to the group of units:

$$
\mathcal{G}=\left\{g \in \mathcal{H}^{0} \mid \text { exists } g^{-1} \in \mathcal{H}^{0} \text { such that } g \bullet g^{-1}=g^{-1} \bullet g=1\right\}
$$

One has the standard transformation rule for the 'covariant differential' $d_{\phi}$ :

$$
d_{\phi}\left(g^{-1} \bullet u \bullet g\right)=g^{-1} \bullet d_{\phi^{g}} \bullet g \Leftrightarrow d_{\phi}\left(A d_{g^{-1}} u\right)=A d_{g^{-1}}\left(d_{\phi^{g}} u\right)
$$

for $u \in \mathcal{H}$, where $A d_{g}(u):=g \bullet u \bullet g^{-1}$. The moduli space results upon dividing the space $\mathcal{S}$ of solutions to (2.12) through the gauge symmetries (2.13). This is a standard Maurer-Cartan problem.

In general, the the action (2.10) is invariant only under 'small' gauge transformations [2], i.e. those transformations for which $g$ can be written in the form:

$$
g=e_{\bullet}^{\alpha}:=\sum_{k \geq 0} \frac{1}{k !} \alpha^{\bullet k}
$$

where $\alpha^{\bullet k}$ stands for $k$-th iteration of the $\bullet$-product of $\alpha$ with itself (and we define $\left.\alpha^{\bullet 0}:=1\right)$.

For infinitesimal $\alpha$, the gauge transformations (2.13) become:

$$
\phi \rightarrow \phi+\delta_{\alpha} \phi
$$

with $\delta_{\alpha} \phi=-d \alpha-[\phi, \alpha]_{\bullet}$. For small $\phi$ and $\alpha$, the moduli problem reduces to its linearized version:

$$
d \phi=0, \phi \equiv \phi-d \alpha
$$

which describes infinitesimal deformations of the background.

\footnotetext{
${ }^{5}$ One has $d\left(\phi^{g}\right)+\phi^{g} \bullet \phi^{g}=g \bullet(d \phi+\phi \bullet \phi) \bullet g^{-1}$. The object $d \phi+\phi \bullet \phi$ is the curvature of the associated superconnection.
} 
Observation Since graded Chern-Simons theories contain higher rank forms, a complete analysis of their gauge symmetries (with a view toward quantization) requires the Batalin-Vilkovisky formalism. The classical part of this analysis was carried out in [19], while gauge fixing and propagators are discussed in [3]. In the present paper, we will be able to avoid the BV formalism by employing the 'resolvent method' of $[9,10]$. The BV analysis gives the same results (as expected from the general observations of $[18])$.

\subsection{D-brane interpretation of the background}

Our string field theory is formulated around a specific background described by certain collections of graded D-branes. A shift of the background (which can be achieved by use of a solution $\phi$ to $(2.12)$ ) deforms the differential $d$ into $d_{\phi} u=d u+[\phi, u]$. It is easy to check (see $[23,24,2]$ ) that such shifts preserve the relevant properties of $d$. When expanded around the shifted background, the string field action has the same form (2.10) up to the addition of an irrelevant constant [23].

As explained in [23, 24], a general background admits a D-brane interpretation which allows for a systematic description in the language of category theory. This is related to the basic fact [34] that degree one superconnections can be decomposed as the sum of a usual connection and a collection of bundle-valued forms, which can be interpreted as condensates of spacetime fields associated with boundary condition changing operators. The decomposition properties of the boundary product and bilinear form with respect to these fields can then be used to extract the D-brane content of the background $[23,24]$. The D-brane interpretation is different at various points in the moduli space. This identifies our moduli space of vacua with the moduli space of topological D-brane composites $[23,24]$. We refer the reader to the papers just cited for a detailed presentation of this analysis. It was shown on general grounds in [23] (and, for the models discussed here, in [39]) that the collection of D-brane composites describing a background $\phi$ defines a 'differential graded category', part of which can be related to certain enhanced triangulated categories $[20]^{6}$. For the B-model version of the theory (which was considered in [26]), the relevant triangulated category essentially coincides with the derived category of coherent sheaves.

From many points of view, the string field theory description must come before any discussion in terms of triangulated categories. For example, a proper definition of

\footnotetext{
${ }^{6}$ We warn the reader that the description of 'D-brane category dynamics' in [23, 24] is only local, i.e. based on the linearized version (2.18) of the equations of motion; similar limitations apply to the triangulated category picture, which is a consequence of this. This is related to the issue of finding a good global definition for the 'moduli space of a triangulated category', which seems to be best approached at the string field theory level.
} 
deformations seems to require string field data, and possesses a standard (i.e. MaurerCartan) formulation only at the string field level [2].

\section{The semiclassical approximation and generalized Ray-Singer torsion}

We are interested in the partition function $Z$ of (2.10). Fixing a flat superconnection $A$, this can be determined by performing a background perturbation expansion around $A$. It is a standard fact that the semiclassical contribution to this expansion is given (up to the trivial factor $e^{-i \lambda S(A)}$, which we shall ignore) by certain functional determinants, which encode the contribution of the kinetic term. The purpose of this section is to evaluate this approximation in order to extract a certain numerical invariant associated with our models.

\subsection{Hermitian structure}

Our gauge-fixing procedure will require that we pick a Riemannian metric $g$ on $L$ and Hermitian metrics on the bundles $E_{n}$. The last determine a Hermitian metric $g_{\mathbf{E}}$ on $\mathbf{E}$, and thus a Hermitian metric on $\operatorname{End}(\mathbf{E})$, given by:

$$
(\alpha, \beta)=\operatorname{tr}\left(\alpha^{\dagger} \circ \beta\right) \quad \text { for } \quad \alpha, \beta \in \operatorname{End}\left(\mathbf{E}_{p}\right), p \in L
$$

where $\alpha^{\dagger}$ is the Hermitian conjugate of $\alpha$ with respect to $g_{\mathbf{E}}$. On the other hand, $g$ induces a Hermitian metric $(.,$.$) on \Lambda^{*}\left(T^{*} L\right)$ in the standard fashion. This is given by:

$$
(* \bar{\omega}) \wedge \eta=(\omega, \eta) v o l_{g} \quad, \text { for } \quad \omega, \eta \in \Lambda^{*}\left(T_{p}^{*} L\right)
$$

where $v l_{g}$ is the volume form induced by $g$ on $L$ (with respect to the fundamental orientation on $L$ ), while $*$ is the complex linear Hodge operator, which is involutive and satisfies:

$$
r k(* \omega)=3-r k \omega
$$

Combining these, we obtain a Hermitian metric $(., .)_{\mathcal{V}}$ on the bundle $\mathcal{V}=\Lambda^{*}\left(T^{*} L\right) \otimes$ $\operatorname{End}(\mathbf{E})$, which can be described through the relation:

$$
\operatorname{tr}\left(* u^{\dagger} \wedge v\right)=(u, v)_{\mathcal{V}} v_{g} l_{g} \quad \text { for } u, v \in \mathcal{V}_{p} \quad, \quad p \in L
$$

It is clear that $(u, v)_{\mathcal{V}}$ vanishes (on bi-homogeneous elements) unless $\Delta(u)=\Delta(v)$ and $r k u=r k v$ (and thus unless $|u|=|v|$ ). In particular, we have induced scalar products on each of the subbundles $\mathcal{V}^{k}$, which are mutually orthogonal with respect to (3.4). Integration over $L$ gives a Hermitian scalar product on the space $\mathcal{H}=\Gamma(\mathcal{V})$ :

$$
h(u, v)=\int_{L}(u, v) v o l_{g}=\int_{L} \operatorname{tr}\left(* u^{\dagger} \wedge v\right), \text { for } u, v \in \mathcal{H}
$$




\subsubsection{The conjugation operator}

This was discussed in detail [2], so I will be short. Since the bilinear form (2.8) is non-degenerate, there exists a unique antilinear operator $c$ on $\mathcal{H}$ with the property:

$$
h(u, v)=\langle c u, v\rangle=\int_{L} \operatorname{str}[(c u) \bullet v] .
$$

This has the following form on decomposable elements:

$c(\omega \otimes f)=(-1)^{n+\Delta(f)(1+r k \omega)}(* \bar{\omega}) \otimes f^{\dagger} \quad, \quad$ for $\quad \omega \in \Omega^{*}(L) \quad$ and $\quad f \in \operatorname{Hom}\left(E_{m}, E_{n}\right)$.

The conjugation operator satisfies:

$$
|c u|=3-|u|, \quad c^{2}=i d,
$$

and thus obeys the axiomatic framework of [36]. Note that $c$ is anti-unitary:

$$
h(u, v)=h(c v, c u)
$$

In the ungraded case $\left(\mathbf{E}=E_{0}\right), c$ reduces to the antilinear Hodge operator $\bar{*}$, coupled to the bundle $\operatorname{End}(E)$.

\subsubsection{The adjoint of $d$ and the Laplacian}

As mentioned above, the scalar product $h$ satisfies the selection rule:

$$
h(u, v)=0, \quad \text { unless }|u|=|v| .
$$

Considering the Hermitian conjugate $d^{\dagger}$ of $d$ (with respect to $h$ ), we have:

$$
d^{\dagger} u=(-1)^{|u|} c d c u \quad, \quad\left|d^{\dagger} u\right|=|u|-1 \quad, \quad\left(d^{\dagger}\right)^{2}=0 \quad .
$$

Using this operator, one constructs the 'deformed Laplacian' $\Delta=d d^{\dagger}+d^{\dagger} d$, which is an order two elliptic operator on $\mathcal{H}$. We also note the relations:

$$
\begin{aligned}
& c d^{\dagger} u=(-1)^{|u|} d c u, \quad d^{\dagger} c u=(-1)^{|u|+1} c d u \\
& d^{\dagger} d c=c d d^{\dagger} \quad, \quad d d^{\dagger} c=c d^{\dagger} d .
\end{aligned}
$$

\subsection{The spacetime ghost grading}

According to (3.10), we have induced Hermitian scalar products on each of the subspaces $\mathcal{H}^{k}$, which are mutually orthogonal with respect to $h$. For what follows, it will be convenient to use the grading $s(u)=1-|u|$, which in the BV formalism corre-

sponds to the ghost number of the string field theory [19]. We shall use the notation 
$\mathcal{H}(\sigma)=\mathcal{H}^{1-\sigma}$ for the homogeneous subspaces of $\mathcal{H}$ with respect to this grading, and the notation $H_{\sigma}(\mathcal{H})=H^{1-\sigma}(\mathcal{H})$ for the associated components of $H^{*}(\mathcal{H})$ (since the ghost grading is decreased by $d$, this takes the original cochain complex $(\mathcal{H}, d)$ into a chain complex, which is why we use homological notation). With this convention, the string field lies in the subspace $\mathcal{H}(0)$ of vanishing ghost number. Note that:

$$
s(d u)=s(u)-1, \quad s(c u)=-1-s(u), \quad s(c d u)=-s(u) .
$$

In particular, $c d$ induces a well-defined antilinear operator on the physical subspace $\mathcal{H}(0)$.

It is clear from the definition of $c$ that a nonzero element $u \in \mathcal{H}$ always has a nonzero conjugate partner $c u$. Since the number of graded components $E_{n}$ is assumed to be finite, there exists a maximal value of the degree $s$, which we shall denote by $N$. The second relation in (3.13) then shows that the minimal value of $s$ is $-1-N$. Accordingly, the degree $||=.1-s($.$) lies in the interval 1-N \ldots 2+N$.

We also recall the standard decompositions:

$$
\mathcal{H}=K \oplus i m d \oplus i m d^{\dagger} \quad, \quad k e r d=i m d \oplus K \quad, \quad k e r d^{\dagger}=i m d^{\dagger} \oplus K
$$

where $K=\operatorname{ker} \Delta=\operatorname{kerd} \cap k e r d^{\dagger}$. Hodge theory leads to the identification $K \approx H_{d}^{*}(\mathcal{H})$.

\subsection{Complex and real determinants and induced Euclidean scalar products}

In the next subsection, it will be convenient to 'decompose our fields into real components' when performing the path integral. For that purpose, we collect some useful facts and definitions. This is entirely trivial and well-known, but I shall state it explicitly nonetheless.

The complex vector space $\mathcal{H}$ can be viewed as a real vector space upon restriction of the field of scalars. Then the Hermitian product $h$ induces an Euclidean scalar product $(.,):.=$ Reh on $\mathcal{H}$. Given a complex vector subspace $W$ of $\mathcal{H}$, it is easy to check that for any vector $u$ in $\mathcal{H}$, one has the equivalence:

$$
(u, v)=0 \text { for all } v \in W \Leftrightarrow<u, v>=0 \text { for all } v \in W \text {. }
$$

Indeed, if $W$ contains a vector $v$, then it also contains the vector $i v$, and one has $(u, i v)=\operatorname{Reh}(u, i v)=\operatorname{Re}[i h(u, v)]=-\operatorname{Imh}(u, v)$. Relation (3.15) shows that the orthogonal complements of $W$ with respect to $h$ and $(.,$.$) coincide, so we shall use the$ symbol $W^{\perp}$ to denote either.

A complex-linear operator $O$ on a complex vector space $V$ can also be viewed as a real-linear operator on the underlying real vector space. Therefore, one has two notions for the determinant of $O$, namely the complex and real determinant. The first is the 
determinant of $O$ viewed as a complex-linear map, i.e. the determinant of its matrix in a complex-linear basis $e_{\alpha}$ of $V$ :

$$
O e_{\alpha}=a_{\beta \alpha} e_{\beta} \Rightarrow \operatorname{det} O=\operatorname{det}\left(a_{\alpha \beta}\right)
$$

It can be described more geometrically by considering the maximal exterior power $\Lambda^{d} V$ of $V$ viewed as a complex vector space (here $d$ is the complex dimension of $V$ ). Indeed, $O$ induces a linear endomorphism of $\Lambda^{d} V$. Since the latter space is complex one-dimensional, this endomorphism is canonically identified with a complex number, which is the determinant of $O$. In fact, $\Lambda^{d} V$ has a basis given by $e_{1} \wedge \ldots \wedge e_{d}$, and $O\left(e_{1}\right) \wedge \ldots \wedge O\left(e_{d}\right)=\operatorname{det} O e_{1} \wedge \ldots \wedge e_{d}$.

The real determinant arises from a similar construction, where one views $O$ as a real linear operator on the real vector space $V$ (whose real dimension is $2 d$ ). The latter admits the basis $e_{1} \ldots e_{d}, i e_{1} \ldots i e_{d}$, which gives:

$$
\begin{gathered}
O e_{\alpha}=x_{\beta \alpha} e_{\alpha}+y_{\beta \alpha}\left(i e_{\alpha}\right) \\
O\left(i e_{\alpha}\right)=-y_{\beta \alpha} e_{\alpha}+x_{\beta \alpha}\left(i e_{\alpha}\right)
\end{gathered}
$$

where $x_{\alpha \beta}=R e a_{\alpha \beta}$ and $y_{\alpha \beta}=I m a_{\alpha \beta}$. The real determinant $\operatorname{det}_{\mathbf{R}} O$ is the determinant of the resulting real $2 d \times 2 d$ matrix. This can again be identified with the operator induced by $O$ on the real one-dimensional vector space $\Lambda_{\mathbf{R}}^{2 d} V$, where $\Lambda_{\mathbf{R}}$ denotes the real exterior product. One has $O\left(e_{1}\right) \wedge_{\mathbf{R}} \ldots \wedge_{\mathbf{R}} O\left(e_{d}\right) \wedge_{\mathbf{R}} O\left(i e_{1}\right) \wedge_{\mathbf{R}} \ldots \wedge_{\mathbf{R}} O\left(i e_{d}\right)=$ $\operatorname{det}_{R}(O) e_{1} \wedge_{\mathbf{R}} \ldots \wedge_{\mathbf{R}} e_{d} \wedge\left(i e_{1}\right) \wedge_{\mathbf{R}} \ldots \wedge_{\mathbf{R}}\left(i e_{d}\right)$. One can show that:

$$
\operatorname{det}_{\mathbf{R}} O=|\operatorname{det} O|^{2}
$$

a formula which will be used repeatedly in later subsections.

\subsection{The semiclassical partition function in the resolvent formalism}

\subsubsection{The computation}

To compute the semiclassical partition function, we use the resolvent formalism of $[9,10]$, in its generalized version discussed in $[11,12]^{7}$ (see $[14,13,15]$ and $[17,16]$ for related issues). For this, we focus on the kinetic term:

$$
S_{k i n}(\phi)=\operatorname{Re}\langle\phi, d \phi\rangle=\operatorname{Re} \int_{L} \operatorname{str}(\phi \bullet d \phi),
$$

\footnotetext{
${ }^{7}$ A streamlined presentation of this material can be found in the thesis of D. H. Adams. The author thanks him for bringing this thesis to his attention.
} 
where $d:=d_{A}$ is the differential on $\mathcal{H}$ associated with the background $A$. The semiclassical partition function is formally given by:

$$
Z_{s c l}=\int \mathcal{D}[\phi] e^{-i \lambda S_{k i n}(\phi)}
$$

where $\lambda>0$ is the coupling constant. This path integral is of course ill-defined. The most severe problem is the presence of zero modes, which are related to the gauge invariance $\phi \rightarrow \phi+d \omega$, with $\omega$ an element of $\mathcal{H}(1)=\mathcal{H}^{0}=\oplus_{m, n} \Omega^{m-n}\left(\operatorname{L}, \operatorname{Hom}\left(E_{m}, E_{n}\right)\right)$. A precise characterization of zero modes is as follows. Using the metric induced on $\mathcal{H}(0)$, we write $S_{k i n}$ in the form:

$$
S_{k i n}(\phi)=\operatorname{Re} h(\phi, c d \phi)=(\phi, c d \phi),
$$

where $(.):,=\operatorname{Reh}(.,$.$) is the Euclidean scalar product induced by h$, if we view $\mathcal{H}^{1}=$ $\mathcal{H}(0)$ as a real vector space. The zero modes lie in the kernel of this quadratic functional. Since $(.,$.$) is nondegenerate, this coincides with the kernel of the antilinear operator$ $T_{0}:=c d: \mathcal{H}(0) \rightarrow \mathcal{H}(0)$. In fact, $T_{0}$ becomes a (real-linear) self-adjoint operator, as can be checked by using invariance of $\langle.,$.$\rangle with respect to d$ :

$$
\left(T_{0} u, v\right)=\left(u, T_{0} v\right) \quad \text { for } \quad u, v \in \mathcal{H}(0) \Rightarrow T_{0}^{t}=T_{0}
$$

where ${ }^{t}$ stands for the adjoint with respect to $(.,$.$) . It is also easy to check that:$

$$
\left(\phi, T_{0} \phi\right)=\left(\phi_{M}, T_{0} \phi_{M}\right)
$$

where we decomposed $\phi:=\phi_{M} \oplus \phi_{K}$ with $\phi_{K} \in \operatorname{kerd}=\operatorname{ker} T_{0}$ and $\phi_{M} \in(k e r d)^{\perp}=$ $\left(\operatorname{ker} T_{0}\right)^{\perp}$. If we let $T_{0}^{\prime}$ denote the restriction of $T_{0}$ to the orthogonal complement of its kernel, then formal integration in (3.20) leads to:

$$
Z_{s c l}=\operatorname{vol}\left(k e r\left(T_{0}\right)\right) \int \mathcal{D}\left[\phi_{M}\right] e^{-i \lambda\left(\phi_{M}, T_{0}^{\prime} \phi_{M}\right)}
$$

Both factors in (3.24) are ill-defined, since the first involves the 'volume' of a vector space while the second is a Gaussian integral for a generally indefinite quadratic form. The second problem is solved in standard manner by replacing $T_{0}^{\prime}$ with the strictly positive operator $\left|T_{0}^{\prime}\right|=\sqrt{T_{0}^{\prime} T_{0}^{\prime}}=\sqrt{\left(d^{\dagger} d\right)_{s=0}^{\prime}}$, where the subscript $s=0$ indicates that $d^{\dagger} d$ is restricted to the subspace $\mathcal{H}(0)$. This leads to the expression:

$$
\int \mathcal{D}\left[\phi_{M}\right] e^{-i \lambda\left(\phi_{M}, T_{0}^{\prime} \phi_{M}\right)}=c t \times\left(\operatorname{det}_{\mathbf{R}}\left|T_{0}^{\prime}\right|\right)^{-1 / 2}
$$

where $\operatorname{det}_{\mathbf{R}}\left|T_{0}^{\prime}\right|$ denotes the real determinant of $\left|T_{0}^{\prime}\right|$. Using $\operatorname{det}_{\mathbf{R}}\left|T_{0}^{\prime}\right|^{-1 / 2}=\operatorname{det}\left|T_{0}^{\prime}\right|^{-1}=$ $\left(\operatorname{det}_{s=0}^{\prime}\left(d^{\dagger} d\right)\right)^{-1 / 2}$, we obtain:

$$
Z_{s c l}=c t \times \operatorname{vol}\left(k e r\left(T_{0}\right)\right)\left(\operatorname{det}_{s=0}^{\prime} d^{\dagger} d\right)^{-1 / 2}
$$


where, for a Hermitian operator $O, \operatorname{det}^{\prime} O$ denotes the complex determinant of $\left.O\right|_{(k e r O)^{\perp}}$.

The last factor of (3.26) is still ill-defined, since it involves an infinite product of eigenvalues. This is a standard problem, which is solved by zeta-function regularization. We remind the reader that, given a positive elliptic operator $O$, acting on sections of a vector bundle over $L$, its zeta function $\zeta_{O}(z)(z \in \mathbf{C})$ is defined as follows. For sufficiently large $\operatorname{Rez}, \zeta_{O}(z)$ is given by the expansion:

$$
\zeta_{O}(z)=\sum_{\lambda} \frac{n_{\lambda}}{\lambda^{z}}
$$

where the sum is over the (strictly positive) eigenvalues $\lambda$ of $O$, whose multiplicities we denote by $n_{\lambda}$. The analytic continuation of this series to the complex plane is meromorphic and regular at the origin. This allows one to define the regularized determinant through the expression: $\operatorname{det}^{r e g}(O):=e^{-\zeta_{O}^{\prime}(0)}$, where $\zeta_{O}^{\prime}(z):=\frac{d}{d z} \zeta_{O}(z)$. Applying this to the operator $\left|T_{0}^{\prime}\right|^{2}$, we obtain:

$$
\operatorname{det}_{s=0}^{\prime, r e g}\left(d^{\dagger} d\right)=e^{-\zeta_{\left(d^{\dagger} d\right)_{s=0}^{\prime}}^{\prime}(0)}
$$

To regularize the volume factor in (3.26), we use the so-called method of resolvents $[9,11,12]$, which proceeds as follows ${ }^{8}$. First, we notice that the kinetic operator $T_{0}=c d$ admits an elliptic resolvent. More precisely, one has the complex of real vector spaces:

$$
(\mathcal{R}) \quad: \quad 0 \rightarrow \mathcal{H}(N) \stackrel{T_{N}=d}{\longrightarrow} \ldots \stackrel{T_{2}=d}{\longrightarrow} \mathcal{H}(1) \stackrel{T_{1}=d}{\longrightarrow} \mathcal{H}(0) \stackrel{T_{0}=c d}{\longrightarrow} \mathcal{H}(0)
$$

(where $N$ denotes the maximal value of the spacetime ghost degree, as discussed in Subsection 3.2), and the associated Laplace operators:

$$
\Delta_{\sigma}:=T_{\sigma}^{t} T_{\sigma}+T_{\sigma+1} T_{\sigma+1}^{t}=d^{\dagger} d+d d^{\dagger}: \mathcal{H}(\sigma) \rightarrow \mathcal{H}(\sigma)
$$

(with $T_{N+1}:=0$ ) are elliptic for all $\sigma=0 \ldots N$. To arrive at (3.30), we used the fact that $T_{\sigma}^{t}=T_{\sigma}^{\dagger}$ for $\sigma>0$ and $T_{0}^{t} T_{0}=d^{\dagger} d$ for $\sigma=0$. Notice that $\mathcal{H}(\sigma)=\mathcal{H}^{1-\sigma}=\Gamma\left(\mathcal{V}^{1-\sigma}\right)$ are in fact complex vector spaces, but the last map in (3.29) is complex antilinear, rather than complex linear. This is why we can only view (3.29) as a complex of real-linear maps.

To regularize $\operatorname{vol}\left(k e r T_{0}\right)$, one picks auxiliary Hermitian metrics $h_{\sigma}^{H}$ on the homology spaces $H_{\sigma}(\mathcal{R})=\operatorname{ker} T_{\sigma} / i m T_{\sigma+1}=H_{d}^{1-\sigma}(\mathcal{H})$ of the resolvent, and considers the Hodge isomorphisms:

$$
f_{\sigma}: K(\sigma)=K^{1-\sigma}=\operatorname{ker} T_{\sigma} \cap \operatorname{ker} T_{\sigma}^{t} \stackrel{\approx}{\longrightarrow} H_{\sigma}(\mathcal{R})=H_{d}^{1-\sigma}(\mathcal{H})
$$

\footnotetext{
${ }^{8}$ Since we work with complex fields, (i.e. $\phi$ varies in a complex vector space) we will have to make some minor modifications in order to adapt the work of [12].
} 
induced by the obvious projections $\operatorname{ker} T_{\sigma} \rightarrow H_{\sigma}(\mathcal{R})$. Given this data, one can formally compute [12]:

$$
\operatorname{vol}\left(\operatorname{ker} T_{0}\right)=\prod_{\sigma=0}^{N}\left[\left(\frac{\operatorname{det}_{\mathbf{R}}^{\prime}\left(T_{\sigma+1}^{t} T_{\sigma+1}\right)}{\operatorname{det}_{\mathbf{R}}\left(f_{\sigma}^{t} f_{\sigma}\right)}\right)^{1 / 2} \operatorname{vol}\left(H_{\sigma}(\mathcal{R})\right) \operatorname{vol}(\mathcal{H}(\sigma+1))\right]^{(-1)^{\sigma}}
$$

where $\operatorname{det}_{\mathbf{R}}$ denotes the determinant of real-linear maps and we defined $\operatorname{det}_{\mathbf{R}}^{\prime}\left(T_{N+1}^{t} T_{N+1}\right):=$ 1 and $\operatorname{vol}(\mathcal{H}(N+1)):=1$. Relation (3.32) gives:

$$
\operatorname{vol}\left(\operatorname{ker} T_{0}\right)=\prod_{\sigma=0}^{N}\left[\frac{\operatorname{det}_{s=\sigma+1}^{\prime}\left(d^{\dagger} d\right)}{\operatorname{det}\left(f_{\sigma}^{\dagger} f_{\sigma}\right)} \operatorname{vol}\left(H_{\sigma}(\mathcal{R})\right) \operatorname{vol}(\mathcal{H}(\sigma+1))\right]^{(-1)^{\sigma}}
$$

(where again we define $\operatorname{det}_{s=N+1}^{\prime}\left(d^{\dagger} d\right):=1$ ). This expression is given a meaning by dropping the ill-defined factors $\operatorname{vol}\left(H_{\sigma}(\mathcal{R})\right)$ and $\operatorname{vol}(\mathcal{H}(\sigma))$ and using zeta-function regularization for the determinants of the positive operators $\left(d^{\dagger} d\right)_{s=\sigma}^{\prime}=\left[\left.\left(d^{\dagger} d\right)\right|_{k e r(d)^{\perp}}\right]_{s=\sigma}$ :

$$
\operatorname{vol}^{r e g}\left(k e r T_{0}\right)=\prod_{\sigma=0}^{N}\left[\frac{\operatorname{det}_{s=\sigma+1}^{\prime}, r e g}{\operatorname{det}\left(f_{\sigma}^{\dagger} f_{\sigma}\right)}\right]^{(-1)^{\sigma}}
$$

Combining with (3.26), we conclude:

$$
\begin{aligned}
& Z_{s c l}=C \operatorname{det}_{s=0}^{\prime, r e g}\left(d^{\dagger} d\right)^{-1 / 2} \prod_{\sigma=0}^{N}\left[\frac{\operatorname{det}_{s=\sigma+1}^{\prime, r e g}\left(d^{\dagger} d\right)}{\operatorname{det}\left(f_{\sigma}^{\dagger} f_{\sigma}\right)}\right]^{(-1)^{\sigma}}=
\end{aligned}
$$



where $C$ is a complex constant. If one uses the normalization conventions of [12], then $C$ can be written as:

$$
C=\left(\frac{\pi}{\lambda}\right)^{\zeta_{\left|T_{0}\right|}(0) / 2} e^{-\frac{i \pi}{4} \eta_{T_{0}}(0)}
$$

where $\zeta_{\left|T_{0}\right|}:=\zeta_{\left|T_{0}^{\prime}\right|}$ is the zeta-function of the positive operator $\left|T_{0}^{\prime}\right|$ and $\eta_{T_{0}}:=\eta_{c d_{s=0}}$ is the eta-function of $T_{0}$. The latter is defined through analytic continuation of the following expression ${ }^{9}$ (which is valid for Rez $>>0$ ):

$$
\eta_{T_{0}}(z)=\sum_{\lambda>0} \frac{n_{\lambda}}{\lambda^{z}}-\sum_{\lambda<0} \frac{n_{\lambda}}{|\lambda|^{z}}
$$

\footnotetext{
${ }^{9}$ Remember that $T_{0}$ is self-adjoint only as a real-linear operator. To find its eigenvalues, one must of course consider the complexification $W$ of the underlying real vector space of $\mathcal{H}(0)$. The complex dimension of $W$ equals $2 \operatorname{dim}_{\mathbf{C}} \mathcal{H}(0)$. The selfadjoint operator $T_{0}$ induces a complex-linear Hermitian operator $T_{0}^{\mathbf{C}}$ on $W$. It is the real eigenvalues of this operator which appear in (3.37) for our case.
} 
the sums being over the strictly positive and strictly negative eigenvalues of $T_{0}\left(n_{\lambda}\right.$ are the multiplicities). We refer the reader to [12] for the justification of (3.36) (in particular, it is shown there that $\eta_{T_{0}}(0)$ is well-defined). Here we only note that the absolute value of $C$ produced by a putative non-perturbative solution of the full graded Chern-Simons theory need not strictly agree with (3.36), due to corrections from a possibly nontrivial gauge stabilizer of the background superconnection $A$ (this is similar to what happens for the ungraded case $[12,13,14,15])$. Therefore, the modulus of expression (3.36) should not be taken at face value.

\subsubsection{Generalized Ray-Singer torsion}

Let us write the partition function (3.35) in an alternate form. First we introduce the notation $\zeta_{\sigma}(z):=\zeta_{\left(d^{\dagger} d\right)_{s=\sigma}^{\prime}}(z)$. Lemma 3.1 of $[12]$ establishes the relation:

$$
\zeta_{\sigma}(z)+\zeta_{\sigma+1}(z)=\zeta_{\Delta_{\sigma}}(z) \quad(\sigma \geq 0)
$$

where $\zeta_{\Delta_{\sigma}}$ is a shorthand for $\zeta_{\Delta_{\sigma}^{\prime}}$. This follows from the fact that $\Delta_{\sigma}^{\prime}=\left(d d^{\dagger}\right)^{\prime} \oplus\left(d^{\dagger} d\right)^{\prime}$ (since $\left.(k e r \Delta)^{\perp}=i m d^{\dagger} \oplus i m d=(k e r d)^{\perp} \oplus\left(k e r d^{\dagger}\right)^{\perp}=k e r\left(d^{\dagger} d\right)^{\perp} \oplus\left(k e r d d^{\dagger}\right)^{\perp}\right)$, which implies $\zeta_{\Delta_{\sigma}^{\prime}}(z)=\zeta_{\left(d^{\dagger} d\right)_{\sigma}^{\prime}}(z)+\zeta_{\left(d d^{\dagger}\right)_{\sigma}^{\prime}}(z)$. Then one notices that $d$ gives an isomorphism between $\operatorname{ker}\left(d^{\dagger} d\right)^{\perp}=\operatorname{ker}(d)^{\perp}$ and $k e r\left(d d^{\dagger}\right)^{\perp}=k e r\left(d^{\dagger}\right)^{\perp}=i m(d)$, thereby inducing a bijection between the nonzero eigenvalues of $\left(d^{\dagger} d\right)_{\sigma+1}$ and $\left(d d^{\dagger}\right)_{\sigma}$ (if $d^{\dagger} d u=\lambda u$ then $d d^{\dagger} d u=\lambda d u$ etc.). Therefore, $\zeta_{\left(d d^{\dagger}\right)_{\sigma}^{\prime}}(z)=\zeta_{\sigma+1}(z)$, which leads to (3.38).

Equation (3.38) implies:

$$
\zeta_{0}+2 \sum_{\sigma=1}^{N}(-1)^{\sigma} \zeta_{\sigma}=\sum_{\sigma=0}^{N}(-1)^{\sigma}(1+2 \sigma) \zeta_{\Delta_{\sigma}}
$$

The next step is to notice that the last two equations in (3.12) imply $\Delta c=c \Delta$ and thus:

$$
c \Delta_{\sigma}=\Delta_{-1-\sigma} c, \Delta_{\sigma} c=c \Delta_{-1-\sigma},
$$

because $s(c u)=-1-s(u)$. Since $c$ is a bijection, this shows that the operators $\Delta_{\sigma}$ and $\Delta_{-1-\sigma}$ are iso-spectral (i.e. have the same eigenvalues, with the same multiplicities). Therefore:

$$
\zeta_{\Delta_{\sigma}}(z)=\zeta_{\Delta_{-1-\sigma}}(z) \quad \text { for } \sigma \in\{-1-N \ldots N\}
$$

Thus:

$$
\sum_{\sigma=0}^{N}(-1)^{\sigma}(1+2 \sigma) \zeta_{\Delta_{\sigma}}=\sum_{\sigma=-N-1}^{N}(-1)^{\sigma} \sigma \zeta_{\Delta_{\sigma}}=\sum_{q=1-N}^{N+2}(-1)^{q} q \zeta_{\Delta^{(q)}}
$$


where $\Delta^{(q)}=\Delta_{1-q}$ is the Laplacian on the space $\mathcal{H}^{q}=\mathcal{H}(1-q)$. Combining (3.39) and (3.42), we obtain:

$$
\frac{1}{2} \zeta_{0}+\sum_{\sigma=1}^{N}(-1)^{\sigma} \zeta_{\sigma}=\frac{1}{2} \sum_{\sigma=-N-1}^{N}(-1)^{\sigma} \sigma \zeta_{\Delta_{\sigma}}=\frac{1}{2} \sum_{q=1-N}^{N+2}(-1)^{q} q \zeta_{\Delta(q)}
$$

where $\sigma$ and $q$ in the right hand side run over their maximal domains of definition $\{-1-N \ldots N\}$ and $\{1-N \ldots N+2\}$. Let us define the quantity:

$T(L, A):=\prod_{q=1-N}^{N+2}\left[\operatorname{det}^{\prime}, r e g \Delta^{(q)}\right]^{\frac{(-1)^{q} q}{2}}=e^{-\frac{1}{2} \sum_{q=1-N}^{N+2}(-1)^{q} q \zeta_{\Delta}^{\prime}(q)}(0)=e^{-\frac{1}{2} \sum_{\sigma=-N-1}^{N}(-1)^{\sigma} \sigma \zeta_{\Delta_{\sigma}}^{\prime}(0)}$

which is a generalized version of the analytic torsion of Ray and Singer $[5,6,7]$. Then:

$$
Z_{s c l}=C T(L, A)^{-1} \prod_{\sigma \geq 0}\left[\operatorname{det}\left(f_{\sigma}^{\dagger} f_{\sigma}\right)^{(-1)^{\sigma+1}}\right]
$$

It is convenient to write $Z_{s c l}=C \tilde{Z}_{s c l}$, where:

$$
\tilde{Z}_{s c l}=T(L, A)^{-1} \prod_{\sigma \geq 0}\left[\operatorname{det}\left(f_{\sigma}^{\dagger} f_{\sigma}\right)^{(-1)^{\sigma+1}}\right]
$$

Observation Relations (3.12) also imply that the operators $\left.\left(d^{\dagger} d\right)_{\sigma}\right|_{k e r(d) \perp}$ and $\left.\left(d d^{\dagger}\right)_{-1-\sigma}\right|_{k e r\left(d^{\dagger}\right)^{\perp}}$ are iso-spectral. Since the latter is iso-spectral with $\left.\left(d^{\dagger} d\right)_{-\sigma}\right|_{(k e r d)^{\perp}}$, this gives the relation:

$$
\zeta_{\sigma}(z)=\zeta_{-\sigma}(z)
$$

which implies $\frac{1}{2} \zeta_{0}+\sum_{\sigma=1}^{N}(-1)^{\sigma} \zeta_{\sigma}=\frac{1}{2} \sum_{\sigma=-N}^{N}(-1)^{\sigma} \zeta_{\sigma}$. Together with (3.43), this gives:

$$
\frac{1}{2} \sum_{q=1-N}^{N+2}(-1)^{q} q \zeta_{\Delta(q)}=\frac{1}{2} \sum_{\sigma=-N}^{N}(-1)^{\sigma} \zeta_{\sigma}
$$

and leads to the following expression for the torsion:

$$
T(L, A)=e^{-\frac{1}{2} \sum_{\sigma=-N}^{N}(-1)^{\sigma} \zeta_{\sigma}^{\prime}(0)} .
$$

Note that the sum in the exponent does not contain a term with $\sigma=-N-1$.

\subsubsection{A Hermitian analogue of the Ray-Singer metric}

The factor $J:=\prod_{\sigma \geq 0}\left[\operatorname{det}\left(f_{\sigma}^{\dagger} f_{\sigma}\right)^{(-1)^{\sigma+1}}\right]$ can be described geometrically as follows. We first notice that there exists a unique antilinear involution $c_{*}: H_{\sigma}(\mathcal{R}) \rightarrow H_{-1-\sigma}(\mathcal{R})$ with the property:

$$
f_{\sigma} c=c_{*} f_{-1-\sigma}
$$


where we consider the Hodge isomorphisms (3.31) for all $\sigma=-N-1 \ldots N$. Given this involution, we use the metrics $h_{\sigma}^{H}$ for $\sigma \geq 0$ to introduce metrics on the spaces $H_{\sigma}(\mathcal{R})=H^{1-\sigma}(\mathcal{H})$ with $\sigma<0$ through the relations:

$$
h_{\sigma}^{H}(\alpha, \beta)=h_{-1-\sigma}^{H}\left(c_{*} \beta, c_{*} \alpha\right) \text { for all } \sigma=-N-1 \ldots-1 .
$$

With respect to these metrics, the operators $c_{*}$ are anti-unitary. Use of particular extension of the auxiliary data $h_{\sigma}^{H}(\sigma \geq 0)$ to the entire cohomology $H_{d}^{*}(\mathcal{H})$ is crucial for the validity of certain arguments below.

We have $f_{\sigma}^{\dagger} f_{\sigma}=c f_{-1-\sigma}^{\dagger} f_{-1-\sigma} c$, which implies:

$$
\operatorname{det}\left(f_{\sigma}^{\dagger} f_{\sigma}\right)=\operatorname{det}\left(f_{-1-\sigma}^{\dagger} f_{-1-\sigma}\right) \text { for all } \sigma \text {, }
$$

where we used $c^{2}=i d$. This allows us to write:

$$
J=\prod_{\sigma \geq 0}\left[\operatorname{det}\left(f_{\sigma}^{\dagger} f_{\sigma}\right)^{(-1)^{1-\sigma}}\right]=\prod_{\sigma=-N-1}^{N}\left(\operatorname{det} f_{\sigma}^{\dagger} f_{\sigma}\right)^{\frac{(-1)^{1-\sigma}}{2}}=\prod_{q=1-N}^{2+N} \operatorname{det}\left(f^{(q) \dagger} f^{(q)}\right)^{\frac{(-1)^{q}}{2}},
$$

where we defined $f^{(q)}:=f_{1-q}: K^{q} \rightarrow H_{d}^{q}(\mathcal{H})$.

Defining $b_{\sigma}:=\operatorname{dim}_{\mathbf{C}} H_{\sigma}(\mathcal{R})$ and $b^{q}:=\operatorname{dim}_{\mathbf{C}} H^{q}(\mathcal{H})$ (so that $b_{\sigma}:=b^{1-\sigma}$ ), let us consider the 'complex determinant line':

$$
\mathcal{L}=\operatorname{det} H_{d}^{*}(\mathcal{H})=\otimes_{\sigma=-N-1}^{N} \Lambda^{b_{\sigma}}\left[H_{\sigma}(\mathcal{R})^{*(1-\sigma)}\right]=\otimes_{q=1-N}^{2+N} \Lambda^{b^{q}}\left[H_{d}^{q}(\mathcal{H})^{* q}\right]
$$

which is a one-dimensional complex vector space. This space carries two Hermitian metrics. The first metric is induced by $g$ and $g_{\mathbf{E}}$ and arises upon transporting the restriction of $h$ to the harmonic subspaces $K(\sigma)$ to metrics $h_{\sigma}=h^{(1-\sigma)}$ on $H_{\sigma}(\mathcal{H})=$ $H_{d}^{1-\sigma}(\mathcal{H})$ through the Hodge isomorphisms $f_{\sigma}: K(\sigma) \rightarrow H_{d}^{1-\sigma}(\mathcal{H})$ :

$h_{\sigma}(u, v)=h\left(f_{\sigma}^{-1} u, f_{\sigma}^{-1} v\right)=h_{\sigma}^{H}\left(\left(f_{\sigma}^{-1}\right)^{\dagger} f_{\sigma}^{-1} u, v\right)=h_{\sigma}^{H}\left(\left(f_{\sigma} f_{\sigma}^{\dagger}\right)^{-1} u, v\right)$ for $u, v \in H_{d}^{1-\sigma}(\mathcal{H})$.

The metrics (3.55) induce a metric $h_{\mathcal{L}}$ on $\mathcal{L}$ known as the Hermitian $L_{2}$-metric. If $e_{\alpha}^{(q)}$ are bases for the complex vector spaces $H_{d}^{q}(\mathcal{H})$, then $e:=\otimes_{q}\left(e_{1}^{(q)} \wedge \ldots \wedge e_{b^{q}}^{(q)}\right)^{* q}$ is a complex basis of $\mathcal{L}$, whose squared norm in the metric $h_{\mathcal{L}}$ is given by:

$$
\|e\|_{\mathcal{L}}^{2}=h_{\mathcal{L}}(e, e)=\prod_{q}\left[\operatorname{det} G^{(q)}\right]^{(-1)^{q}}
$$

where $G_{\alpha \beta}^{(q)}=h^{(q)}\left(e_{\alpha}^{(q)}, e_{\beta}^{(q)}\right)$ are the (positive-definite) Hermitian Gramm matrices. On the other hand, the auxiliary metrics $h_{\sigma}^{H}=h_{H}^{(1-\sigma)}$ on $H_{\sigma}(\mathcal{H})=H 1-\sigma_{d}(\mathcal{H})$ induce a metric $h_{\mathcal{L}}^{H}$ on $\mathcal{L}$, for which:

$$
\left(\|e\|_{\mathcal{L}}^{H}\right)^{2}=h_{\mathcal{L}}^{H}(e, e)=\prod_{q}\left[\operatorname{det} G_{H}^{(q)}\right]^{(-1)^{q}}
$$


with $\left(G_{H}^{(q)}\right)_{\alpha \beta}=h_{H}^{(q)}\left(e_{\alpha}^{(q)}, e_{\beta}^{(q)}\right)$. It is clear from this description and from (3.53), (3.55) that the two norms on $\mathcal{L}$ are related through:

$$
\|.\|_{\mathcal{L}}^{H}=J\left\|_{\|}\right\|_{\mathcal{L}}
$$

Let us consider the quantity:

$$
\|.\|_{R S}=T(L, A)\left\|_{.}\right\|_{\mathcal{L}}
$$

which is a norm on $\mathcal{L}$ generalizing the standard Ray-Singer norm [1]. This norm is independent of $h_{\sigma}^{H}$. With this definition, one can write $\tilde{Z}_{s c l}$ as:

$$
\tilde{Z}_{s c l}=\frac{\|\cdot\|_{\mathcal{L}}^{H}}{\|\cdot\|_{R S}}
$$

which displays the dependence of $\tilde{Z}_{s c l}$ on the auxiliary data $h_{\sigma}^{H}$.

\subsubsection{Metric induced on the real determinant line}

The traditional formulation of Ray-Singer torsion in the ungraded case involves connections defined on real vector bundles. As a consequence, one obtains a norm defined not on the complex determinant line but on its real analogue. Since we work with complex vector bundles and connections, it is more natural in our case to use the metric of the previous subsection, which is defined on the complex determinant line. Here we explain the relation between this formulation and the traditional description.

Let us define the real determinant line:

$$
\Lambda:=\otimes_{\sigma=-N-1}^{N} \Lambda_{\mathbf{R}}^{2 b_{\sigma}}\left[H_{\sigma}(\mathcal{R})^{*(1-\sigma)}\right]=\otimes_{q=1-N}^{2+N} \Lambda_{\mathbf{R}}^{2 b^{q}}\left[H_{d}^{q}(\mathcal{H})^{* q}\right]
$$

where all antisymmetrized and tensor products are now taken over the field of real numbers. There exists a natural isomorphism between $\Lambda$ and the second exterior power $\Lambda_{\mathbf{R}}^{2} \mathcal{L}$ of the complex line, where $\mathcal{L}$ is viewed as a real two-dimensional vector space by restriction of the field of scalars. This isomorphism can be described as follows. Given bases $e_{1}^{(q)} \ldots e_{b^{q}}^{(q)}$ of the complex vector spaces $H^{q}(\mathcal{H})$, consider the bases $e_{1}^{(q)} \ldots e_{b^{q}}^{(q)}, i e_{1}^{(q)} \ldots i e_{b^{q}}^{(q)}$ of the underlying real vector spaces, as well as the associated bases $e:=\otimes_{q}\left(e_{1} \wedge \ldots \wedge e_{b^{q}}\right)^{* q}$ and $\epsilon:=\otimes_{q}\left(e_{1} \wedge_{\mathbf{R}} \ldots \wedge_{\mathbf{R}} e_{b^{q}} \wedge_{\mathbf{R}}\left(i e_{1}\right) \wedge_{\mathbf{R}} \ldots \wedge_{\mathbf{R}}\left(i e_{b^{q}}\right)\right)^{* q}$ of $\mathcal{L}$ and $\Lambda$. Under a change $e_{i}^{(q)} \rightarrow e_{i}^{\prime(q)}=M_{q} e_{i}$ of the bases $e_{1}^{(q)} \ldots e_{b^{q}}^{(q)}$ (where $M_{q}$ are invertible complex- linear operators in $H^{q}(\mathcal{H})$ ), we have:

$$
\begin{aligned}
& e^{\prime}:=\otimes_{q}\left(e_{q}^{\prime} \wedge \ldots \wedge e_{q}^{\prime}\right)^{* q}=\prod_{q}\left(\operatorname{det} M_{q}\right)^{(-1)^{q}} e \\
& \epsilon^{\prime}:=\otimes_{q}\left(e_{1}^{\prime} \wedge_{\mathbf{R}} \ldots \wedge_{R} e_{b^{q}}^{\prime} \wedge_{\mathbf{R}}^{\prime}\left(i e_{1}^{\prime}\right) \wedge_{\mathbf{R}} \ldots \wedge_{\mathbf{R}}\left(i e_{b^{q}}^{\prime}\right)\right)^{* q}=\left|\prod_{q} \operatorname{det} M_{q}^{(-1)^{q}}\right|^{2} \epsilon .
\end{aligned}
$$


Considering the basis $e$, ie of the real vector space $\mathcal{L}$, we obtain a basis $\epsilon_{0}:=e \wedge_{\mathbf{R}}(i e)$ of $\Lambda_{\mathbf{R}}^{2} \mathcal{L}$, which transforms as follows:

$$
\epsilon_{0}^{\prime}:=e^{\prime} \wedge_{\mathbf{R}}\left(i e^{\prime}\right)=\left|\prod_{q}\left(\operatorname{det} M_{q}\right)^{(-1)^{q}}\right|^{2} \epsilon_{0}
$$

The isomorphism $\phi: \Lambda_{\mathbf{R}}^{2} \mathcal{L} \rightarrow \Lambda$ is obtained by taking $\epsilon_{0}$ into $\epsilon$. This is well-defined because $\epsilon$ and $\epsilon_{0}$ have the same transformation rules. Note that both of the real lines $\Lambda$ and $\Lambda_{\mathbf{R}}^{2} \mathcal{L}$ are equipped with orientations induced from the complex structure of $H^{q}(\mathcal{H})$, and the vectors $\epsilon_{0}$ and $\epsilon$ always agree with these orientations.

Given the metrics $g$ on $L$ and $g_{\mathbf{E}}$ on $\mathbf{E}$, we consider these constructions for bases $e_{1} \ldots e_{b^{q}}$ which are orthonormal with respect to the Hermitian metrics $h^{q}=h_{1-q}$ which induced (through $f_{\sigma}$ ) on $H^{q}(\mathcal{H})$. In this case, only unitary transformations $M_{q}$ are allowed, hence $\epsilon_{0}$ and $\epsilon$ are uniquely determined by the metric data (since $\left|\operatorname{det} M_{q}\right|=$ 1 ), while $e$ is determined up to multiplication by the phase factor $\prod_{q} \operatorname{det} M_{q}^{(-1)^{q}}$. By construction, the Hermitian $L_{2}$ metric on $\mathcal{L}$ is uniquely determined by the constraint ${ }^{10}$ :

$$
\|e\|_{\mathcal{L}}=1
$$

This induces a Euclidean metric $(., .)_{\mathcal{L}}:=\operatorname{Re}_{\mathcal{L}}(.,$.$) on the underlying real vector space,$ which makes $(e, i e)$ into a real orthonormal basis of $\mathcal{L}$. Upon taking the second exterior power, we have an induced Euclidean metric (.,.) on $\Lambda_{\mathbf{R}}^{2} \mathcal{L}$, which is uniquely determined by the constraint:

$$
\left\|\epsilon_{0}\right\|=1
$$

In fact, given $u=\alpha e$ and $v=\beta e$ in $\mathcal{L}$, with $\alpha, \beta$ some complex constants, we have $u \wedge_{\mathbf{R}} v=\operatorname{Im}(\bar{\alpha} \beta) \epsilon_{0}$ and $\left\|u \wedge_{R} v\right\|=|\operatorname{Im}(\bar{\alpha} \beta)|$.

On the other hand, one has an Euclidean $L_{2}$-metric $(., .)_{\Lambda}$ on $\Lambda$, which is uniquely determined by the condition:

$$
\|\epsilon\|_{\Lambda}=1
$$

This is the metric induced by the Euclidean scalar products $(., .)_{\sigma}:=\operatorname{Reh}_{\sigma}(.,$.$) associ-$ ated with the Hermitian metrics $h_{\sigma}$ on $H_{\sigma}(\mathcal{H})$. Since $\phi$ maps $\epsilon_{0}$ into $\epsilon$, it is clear from (3.65) and (3.66) that $\|\phi(v)\|=\|v\|_{\Lambda}$. Hence the Euclidean $L_{2}$ metric is naturally induced by the Hermitian $L_{2}$ metric via the (metric-independent) isomorphism $\phi$.

Conversely, the Euclidean $L_{2}$ metric determines the vector $\epsilon$ (and thus the vector $\left.\epsilon_{0}\right)$, via relation (3.66). (The apparent sign ambiguity is fixed by the condition that $\epsilon$ must agree with the orientation of $\Lambda$ ). Then $e$ is determined up to a phase factor by

\footnotetext{
${ }^{10}$ Indeed, any element $v$ of $\mathcal{L}$ has the form $u=\alpha e$ with $\alpha$ a complex constant, so relation (3.64) determines $\|v\|=|\alpha|$. Knowledge of the norm on $\mathcal{L}$ then determines the metric in standard fashion.
} 
the relation $e \wedge_{\mathbf{R}}(i e)=\epsilon_{0}$. Since multiplying $e$ by a phase factor does not affect the metric determined by relation $(3.64)$, it follows that $\|.\|_{\mathcal{L}}$ is uniquely determined by the Euclidean $L_{2}$ metric.

We conclude that the Hermitian and Euclidean $L_{2}$ metrics completely determine each other. Which one we use is simply a matter of convention. In this note, we use the metric $\|.\|_{\mathcal{L}}$, which is better adapted to our complex formalism. In the ungraded case $\left(\mathbf{E}=E_{0}\right)$, the Euclidean $L_{2}$ metric arises when considering the complex bundle as a real bundle by forgetting its complex structure.

\subsubsection{Metric-independence of the Ray-Singer norm}

It turns out that the generalized Ray-Singer norm is independent of $g$ and $g_{\mathbf{E}}$, and therefore completely independent of metric data. This statement, which parallels a well-known result for the ungraded case due to Ray and Singer, follows from the general discussion given in [9] and [12]. The results of [12] assure independence of $\|.\|_{R S}$ of all metric data due to the following facts:

(1) The resolvent (3.29) is topological, i.e. the complex

$$
0 \rightarrow \mathcal{H}(N) \stackrel{T_{N}=d}{\longrightarrow} \ldots \stackrel{T_{2}=d}{\longrightarrow} \mathcal{H}(1) \stackrel{T_{1}=d}{\longrightarrow} \operatorname{ker}\left(d_{s=0}\right)=\operatorname{ker}\left(T_{0}\right)
$$

is defined without reference to any metric.

(2) The resolvent is also elliptic, i.e. the deformed Laplacians $\Delta_{\sigma}=T_{\sigma}^{t} T_{\sigma}+$ $T_{\sigma+1} T_{\sigma+1}^{t}=\left(d d^{\dagger}+d^{\dagger} d\right)_{s=\sigma}$ are elliptic operators for all $\sigma=0 \ldots N$.

(3) The base manifold $L$ is odd dimensional.

The proof (which can be found in [12]) involves a combination of results in elliptic operator theory with arguments in linear algebra.

A particularly simple case arises when the background superconnection $A$ is acyclic, i.e. the cohomology $H_{d}^{*}(\mathcal{H})$ vanishes in all degrees. In this situation, the quantity $\tilde{Z}_{\text {scl }}$ coincides with $T(L, A)^{-1}$ and is independent of all metric data. Examples of acyclic backgrounds are provided by condensation of scalars in boundary condition changing sectors of 'topological brane-antibrane pairs', as discussed in detail in [19] and [2].

\subsubsection{The complex prefactor}

Let us turn to the complex prefactor $C$ given in (3.36). A result of [12] implies that:

$$
\zeta_{\left|T_{0}\right|}(0)=-\sum_{\sigma=0}^{N}(-1)^{\sigma} \operatorname{dim} H_{\sigma}(\mathcal{R})=\sum_{q=1-N}^{1}(-1)^{q} \operatorname{dim} H_{d}^{q}(\mathcal{H})
$$


which shows that the absolute value of $C$ is independent of metric data. This shows that $\left|Z_{s c l}\right|$ depends only on the metrics $h_{\sigma}^{H}$.

On the other hand, the value $\eta_{T_{0}}(0)$ may depend on $g$ and $g_{\mathbf{E}}$, which means that the phase factor of the semiclassical partition function will generally carry a metric dependence. This parallels well-known results valid for the ungraded case (standard Chern-Simons field theory) [4].

\section{Example: D-brane pairs of unit relative grade in a scalar background}

Consider a D-brane pair $(a, b)$ such that $\operatorname{grade}(a)=0$ and $\operatorname{grade}(b)=1$. In this case, the underlying graded bundle is $\mathbf{E}=E_{a} \oplus E_{b}$, where $E_{a}$ and $E_{b}$ are the flat bundles describing the reference D-brane background. We let $A_{a}$ and $A_{b}$ be associated flat connections. The space $\mathcal{H}^{q}$ consists of sections of the bundle $\mathcal{V}^{q}=\Lambda^{q}\left(T^{*} L\right) \otimes E n d\left(E_{a}\right) \oplus$ $\Lambda^{q}\left(T^{*} L\right) \otimes \operatorname{End}\left(E_{b}\right) \oplus \Lambda^{q-1}\left(T^{*} L\right) \otimes \operatorname{Hom}\left(E_{a}, E_{b}\right) \oplus \Lambda^{q+1}\left(T^{*} L\right) \otimes \operatorname{Hom}\left(E_{b}, E_{a}\right)$. Such elements can be viewed as matrices of bundle-valued forms:

$$
u=\left[\begin{array}{cc}
u_{q} & u_{q+1} \\
u_{q-1} & \hat{u}_{q}
\end{array}\right] \quad, \text { for }|u|=q,
$$

where the subscript denotes form rank and the bundle components of $u_{q}=u_{a a}, \hat{u}_{q}=$ $u_{b b}, u_{q+1}=u_{b a}$ and $u_{q-1}=u_{a b}$ satisfy $u_{\alpha \beta} \in \Omega^{q+\operatorname{grade}(\alpha)-\operatorname{grade}(\beta)}\left(L, \operatorname{Hom}\left(E_{\alpha}, E_{\beta}\right)\right)$. This corresponds to presenting $u$ as the direct sum:

$$
u=\bigoplus_{\alpha, \beta \in\{a, b\}} u_{\alpha \beta} .
$$

We shall consider backgrounds of the form:

$$
\phi=\left[\begin{array}{cc}
0 & 0 \\
\phi_{0} & 0
\end{array}\right], \quad|\phi|=1
$$

where $\phi_{0}$ is a zero-form valued in the bundle $\operatorname{Hom}\left(E_{a}, E_{b}\right)$. In this case, the equations of motion $d \phi+\phi \bullet \phi=0$ reduce to $d \phi_{0}=0$, which means that $\phi_{0}$ is a covariantlyconstant section of $\operatorname{Hom}\left(E_{a}, E_{b}\right)$. The shifted background is the flat superconnection $A=\left(A_{a} \oplus A_{b}\right)+\phi$. We shall assume that $\phi_{0}$ is a bundle morphism in the restricted sense, i.e. we require ${ }^{11}$ that the rank of the fiber maps $\phi_{0}(p):\left(E_{a}\right)_{p} \rightarrow\left(E_{b}\right)_{p}$ is independent of the point $p \in L$.

\footnotetext{
${ }^{11}$ This assumption allows us to treat the kernel and cokernel of $\phi_{0}$ as subbundles of $E_{a}$ and $E_{b}$. Allowing maps $\phi_{0}$ of non-constant rank leads to situations which are better described in terms of sheaf theory. This is very similar to the case of holomorphic bundles vs. holomorphic sheaves. Note that constancy of $r k \phi_{0}(p)$ is a purely technical assumption - there is no physical reason to restrict to scalar backgrounds of constant rank.
} 
Let us assume that the reference flat bundles $\left(E_{a}, A_{a}\right)$ and $\left(E_{b}, A_{b}\right)$ are unitary, i.e. they admit covariantly-constant Hermitian metrics ${ }^{12}$. We shall pick the metric $g_{\mathbf{E}}$ on E to be induced by two such metrics. With this hypothesis, it was showed in [2] that the deformed Laplacian $\Delta_{\phi}=d_{\phi} d_{\phi}^{\dagger}+d_{\phi}^{\dagger} d_{\phi}$ in the background $\phi_{0}$ has the form:

$$
\Delta_{\phi} u=\left[\begin{array}{cc}
\Delta_{\phi}^{(a a)} u_{q} & \Delta_{\phi}^{(b a)} u_{q+1} \\
\Delta_{\phi}^{(a b)} u_{q-1} & \Delta_{\phi}^{(b b)} \hat{u}_{q}
\end{array}\right]
$$

with:

$$
\Delta_{\phi}^{(\alpha \beta)} u_{\alpha \beta}=\Delta u_{\alpha \beta}+u_{\alpha \beta} \circ t^{(\alpha)}+t^{(\beta)} \circ u_{\alpha \beta}
$$

where we defined:

$$
t^{(a)}=\phi_{0}^{\dagger} \phi_{0} \in \operatorname{End}\left(E_{a}\right) \quad \text { and } \quad t^{(b)}=\phi_{0} \phi_{0}^{\dagger} \in \operatorname{End}\left(E_{b}\right)
$$

and where $\Delta u_{\alpha \beta}$ stands for the form Laplacian coupled to the flat connection induced by $A_{\alpha}$ and $A_{\beta}$ on the bundle $\operatorname{Hom}\left(E_{\alpha}, E_{\beta}\right)$. In direct sum notation, we have:

$$
\Delta_{\phi}=\oplus_{\alpha \beta} \Delta_{\phi}^{(\alpha \beta)}
$$

The fact that the deformed Laplacian decomposes in this manner is a characteristic of scalar background of the particular form (4.3).

Since the subspaces $\Omega^{*}\left(L, \operatorname{Hom}\left(E_{\alpha}, E_{\beta}\right)\right) \subset \mathcal{H}$ are mutually orthogonal with respect to the scalar product $h$, we have:

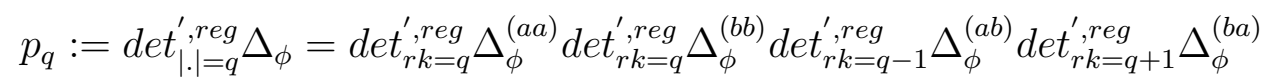

and:

$$
T(L, A)^{2}=\prod_{q=1-N}^{N+2}\left(\text { det }_{|\cdot|{ }^{\prime},{ }^{\prime} \text {, }}^{\text {reg }} \Delta_{\phi}\right)^{q(-1)^{q}}=\prod_{q=1-N}^{N+2} p_{q}^{q(-1)^{q}} .
$$

It was showed in [2] that the kernel $K$ of $\Delta_{\phi}$ coincides (up to a twist of gradings) with the space of harmonic forms valued in the flat bundle $\operatorname{End}\left(R \oplus I^{\perp}\right)$, where $R=$ $\operatorname{ker} \phi$ and $I=i m \phi$ are (flat) subbundles of $E_{a}$ and $E_{b}$ :

$$
K^{q}=\Omega_{\text {harm }}^{q}(L, \operatorname{End}(R)) \oplus \Omega_{\text {harm }}^{q}\left(L, \operatorname{End}\left(I^{\perp}\right)\right) \oplus \Omega_{\text {harm }}^{q-1}\left(L, \operatorname{Hom}\left(R, I^{\perp}\right)\right) \oplus \Omega_{\text {harm }}^{q+1}\left(L, \operatorname{Hom}\left(I^{\perp}, K\right)\right) .
$$

Using the covariantly-constant metrics on $E_{a}$ and $E_{b}$, we identify $I^{\perp}$ with the cokernel $Q$ of the flat bundle map $\phi_{0}: E_{a} \rightarrow E_{b}$ :

$$
I^{\perp} \approx Q:=\operatorname{coker} \phi_{0}=E_{b} / i m \phi_{0}
$$

\footnotetext{
${ }^{12}$ In physical language, this means that we are dealing with unitary connections, i.e. connections whose matrices are anti-Hermitian in appropriate local frames.
} 
Hodge theory leads to identifications $H_{d_{\phi}}^{q}(\mathcal{H})=K^{q}$ and $\Omega_{\text {harm }}^{k}(V) \approx H^{k}(V)$, where $V$ is any of the flat bundles $\operatorname{End}(R), \operatorname{End}\left(I^{\perp}\right), \operatorname{Hom}\left(R, I^{\perp}\right)$ and $\operatorname{Hom}\left(I^{\perp}, R\right)$, while $H^{*}(V)$ stands for the usual cohomology with coefficients in the local system determined by $V$. Combining everything, we obtain:

$$
H_{d_{\phi}}^{q}(\mathcal{H})=H^{q}(L, \operatorname{End}(R)) \oplus H^{q}(L, \operatorname{End}(Q)) \oplus H^{q-1}(L, H o m(R, Q)) \oplus H^{q+1}(L, H o m(Q, R)) .
$$

Using (4.12), we find that the Ray-Singer metric is defined on the determinant line:

$$
\begin{aligned}
\mathcal{L} & =\operatorname{det} H_{d_{\phi}}^{*}(\mathcal{H})=\otimes_{q} \Lambda^{\max } H_{d_{\phi}}^{q}(\mathcal{H})^{* q}= \\
& =\operatorname{det} H^{*}(L, \operatorname{End}(R)) \otimes \operatorname{det} H^{*}(L, \operatorname{End}(Q)) \otimes\left[\operatorname{det} H^{*}(L, H \operatorname{Hom}(R, Q))\right]^{*} \otimes\left[\operatorname{det} H^{*}(L, H o m(Q, R))\right]^{*} .
\end{aligned}
$$

\subsection{The case of trivial flat bundles}

Let us consider the particularly simple case when $E_{a}$ and $E_{b}$ (of ranks $r_{a}$ and $r_{b}$ ) are trivial as flat vector bundles, so that all components $u_{k}, \hat{u}_{k}, u_{k+1}, u_{k-1}$ can be viewed as operator-valued forms, and $d$ coincides with the de Rham differential acting on such forms. In this situation, the condition $d \phi_{0}=0$ means that $\phi_{0}$ is a constant linear operator from $\mathbf{C}^{r_{a}}$ to $\mathbf{C}^{r_{b}}$. Since the flat bundle structure is trivial, we expect to obtain a particularly simple expression for $T(L, A)$. We show below that this expectation is fulfilled.

For this, we first notice that the non-negative operators $t^{(a)}=\phi_{0}^{\dagger} \phi_{0} \in \operatorname{End}\left(\mathbf{C}^{r_{a}}\right)$ and $t^{(b)}=\phi_{0} \phi_{0}^{\dagger} \in \operatorname{End}\left(\mathbf{C}^{r_{b}}\right)$ have the same nonzero eigenvalues. This follows form the following variant of the argument used in Subsection 3.4.2. Noting that $\operatorname{ker}\left(\phi_{0}^{\dagger} \phi_{0}\right)^{\perp}=$ $\operatorname{ker}\left(\phi_{0}\right)^{\perp}$ and $\operatorname{ker}\left(\phi_{0} \phi_{0}^{\dagger}\right)^{\perp}=\operatorname{ker}\left(\phi_{0}^{\dagger}\right)^{\perp}=i m\left(\phi_{0}\right)$, the restriction of $\phi_{0}$ gives an isomorphism:

$$
\phi_{0}: \operatorname{ker}\left(\phi_{0}^{\dagger} \phi_{0}\right)^{\perp} \stackrel{\approx}{\rightarrow} \operatorname{ker}\left(\phi_{0} \phi_{0}^{\dagger}\right)^{\perp}
$$

This induces a bijection between the non vanishing eigenvalues of $\phi_{0}^{\dagger} \phi_{0}$ and $\phi_{0} \phi_{0}^{\dagger}$, since $\phi_{0}^{\dagger} \phi_{0} v=\lambda v$ for some $\lambda \in \mathbf{R}_{+}^{*}$ and $v \in \mathbf{C}^{r_{a}}$ implies $\phi_{0} \phi_{0}^{\dagger}(\phi v)=\lambda \phi_{0} v$. In particular, the ranks of the two operators coincide:

$$
r k\left(\phi_{0}^{\dagger} \phi_{0}\right)=r k\left(\phi_{0} \phi_{0}^{\dagger}\right):=\rho
$$

while their defects are given by:

$$
\operatorname{dim} \operatorname{ker}\left(\phi_{0}^{\dagger} \phi_{0}\right)=r k R=r_{a}-\rho, \operatorname{dim} \operatorname{ker}\left(\phi_{0} \phi_{0}^{\dagger}\right)=r k Q=r_{b}-\rho
$$

Consider unitary transformations $S_{a}$ and $S_{b}$ of $\mathbf{C}^{r_{a}}$ and $\mathbf{C}^{r_{b}}$ which diagonalize $\phi_{0}^{\dagger} \phi_{0}$ and $\phi_{0} \phi_{0}^{\dagger}$ :

$$
\begin{aligned}
& S_{a} \phi_{0}^{\dagger} \phi_{0} S_{a}^{-1}=D^{(a)}:=\operatorname{diag}\left(\lambda_{1}^{(a)} \ldots \lambda_{r_{a}}^{(a)}\right) \\
& S_{b} \phi_{0} \phi_{0}^{\dagger} S_{b}^{-1}=D^{(b)}:=\operatorname{diag}\left(\lambda_{1}^{(b)} \ldots \lambda_{r_{b}}^{(b)}\right)
\end{aligned}
$$


with $\lambda_{i}^{(a)}, \lambda_{j}^{(b)} \geq 0$. In view of the above, we can always pick $S_{a}$ and $S_{b}$ such that:

$$
\begin{aligned}
& \lambda_{i}^{(a)}=\lambda_{i}^{(b)}:=\lambda_{i} \text { for } i=1 \ldots \rho \text { and } \\
& \lambda_{i}^{(a)}=0 \text { for } i=\rho+1 \ldots r_{a} \\
& \lambda_{j}^{(b)}=0 \text { for } j=\rho+1 \ldots r_{b} .
\end{aligned}
$$

It is easy to see that the unitary transformation $S=S_{a} \oplus S_{b} \equiv\left[\begin{array}{cc}S_{a} & 0 \\ 0 & S_{b}\end{array}\right]$ preserves the decomposition (4.4) of $\Delta_{\phi}$ while bringing $\Delta_{\phi}^{(\alpha \beta)}$ to the form:

$$
\Delta_{\phi}^{(\alpha \beta)} u_{\alpha \beta}=\Delta u_{\alpha \beta}+D^{(\beta)} \circ u_{\alpha \beta}+u_{\alpha \beta} \circ D^{(\alpha)}
$$

which act on the matrix components ${ }^{13}$ of $u_{\alpha \beta}$ as:

$$
\Delta_{\phi}^{(\alpha \beta)} u_{\alpha \beta}^{j i}=\Delta u_{\alpha \beta}^{j i}+\left(\lambda_{i}^{(\alpha)}+\lambda_{j}^{(\beta)}\right) u_{\alpha \beta}^{j i}
$$

This leads to the expression:

$$
p_{q}=p_{q}^{(1)} p_{q}^{(2)} p_{q}^{(3)}
$$

where:

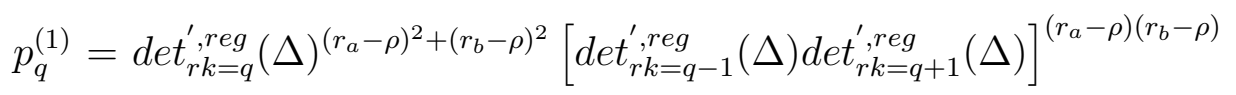

$$
\begin{aligned}
& p_{q}^{(2)}=\prod_{i, j=1}^{\rho}\left[\operatorname{det}_{r k=q}^{\prime, r e g}\left(\Delta+\lambda_{i}+\lambda_{j}\right)\right]^{2} \operatorname{det}_{r k=q-1}^{\prime, r e g}\left(\Delta+\lambda_{i}+\lambda_{j}\right) \operatorname{det}_{r k=q+1}^{\prime, r e g}\left(\Delta+\lambda_{i}+\lambda_{j}\right)
\end{aligned}
$$



It is easy to check that:

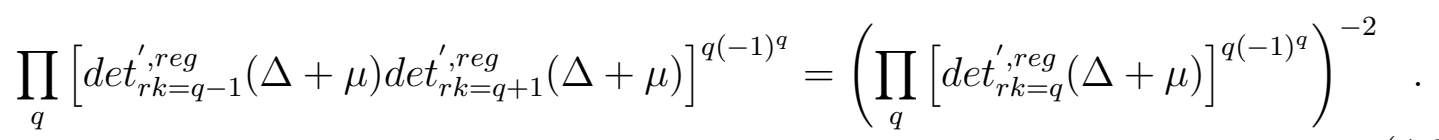

Therefore, one has $\prod_{q}\left(p_{q}^{(2)}\right)^{q(-1)^{q}}=\prod_{q}\left(p_{q}^{(3)}\right)^{q(-1)^{q}}=1$ and we obtain:

$$
T(L, A)=\left[\prod_{q}\left(p_{q}\right)^{q(-1)^{q}}\right]^{1 / 2}=\left[\prod_{q}\left(p_{q}^{(1)}\right)^{q(-1)^{q}}\right]^{1 / 2}=T(L)^{\left(r_{a}-r_{b}\right)^{2}}
$$

\footnotetext{
${ }^{13}$ Picking orthonormal bases $e_{i}^{(a)}$ and $e_{j}^{(b)}$ of $\mathbf{C}^{r_{a}}$ and $\mathbf{C}^{r_{b}}$, we define $u_{\alpha \beta}^{j i}$ through $u_{\alpha \beta}\left(e_{i}^{(\alpha)}\right)=$ $u_{\alpha \beta}^{j i} e_{j}^{(\beta)}$.
} 
where $T(L)$ is the usual Ray-Singer torsion of $L$ (i.e. the standard analytic torsion for the trivial flat complex line bundle $\mathcal{O}_{L}$ over $\left.L\right)$ :

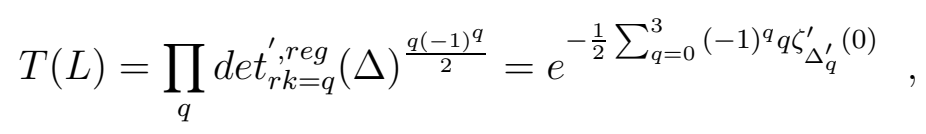

with $\Delta_{q}$ the Laplacian on $\Omega^{q}(L)$. The determinant line can be found by noticing that $R=\mathcal{O}_{L}^{\oplus\left(r_{a}-\rho\right)}$ and $Q=\mathcal{O}_{L}^{\oplus\left(r_{b}-\rho\right)}$, where $\mathcal{O}_{L}$ is the trivial complex flat line bundle over $L$. This implies $\operatorname{End}(R)=\mathcal{O}_{L}^{\oplus\left(r_{a}-\rho\right)^{2}}, \operatorname{End}(Q)=\mathcal{O}_{L}^{\oplus\left(r_{b}-\rho\right)^{2}}$ and $\operatorname{Hom}(R, Q)=$ $\operatorname{Hom}(Q, R)=\mathcal{O}_{L}^{\oplus\left(r_{a}-\rho\right)\left(r_{b}-\rho\right)}$ (as flat line bundles), thereby giving the determinant line:

$$
\mathcal{L}=\operatorname{det} H^{*}\left(L, \mathcal{O}_{L}\right)^{\otimes\left(r_{a}-r_{b}\right)^{2}}=\operatorname{det} H^{*}(L)^{\otimes\left(r_{a}-r_{b}\right)^{2}} .
$$

Combining with (4.24), we see that $T(L, A)$ is the norm induced on $\operatorname{det} H^{*}\left(L, \mathcal{O}_{L}\right)^{\otimes\left(r_{a}-r_{b}\right)^{2}}$ by the usual Ray-Singer norm on $\operatorname{det}^{*}\left(L, \mathcal{O}_{L}\right)=\operatorname{det} H^{*}(L)$ (considered in the 'complex' formalism discussed in Subsection 3.4.3).

Observation If $r_{a}=r_{b}$, then it was showed in $[19,2]$ that a background $\phi_{0}$ which is a flat isomorphism leads to an acyclic composite, i.e. the complex of the shifted differential $d_{\phi}$ is acyclic in all degrees. In this case (and with trivial flat connections $A_{a}$ and $\left.A_{b}\right)$, the generalized Ray-Singer torsion is equal to one.

\section{Acknowledgments}

I thank R. Roiban for comments on the manuscript and Martin Rocek for interest and support. The present work was supported by the Research Foundation under NSF grant PHY-9722101.

\section{References}

[1] J. M. Bismut, W. Zhang, An extension of a theorem of Cheeger and Muller, Asterisque 205 (1992).

[2] C. I. Lazaroiu, R. Roiban, Holomorphic potentials for graded D-branes, hep-th/0110288.

[3] C. I. Lazaroiu, R. Roiban, Holomorphic potentials for graded D-branes, JHEP 02(2002) 038, hep-th/0110288.

[4] E. Witten, Quantum field theory and the Jones polynomial, Commun Math Phys 121 (1989) 351. 
[5] D. B. Ray, Reidemeister torsion and the Laplacian on lens spaces, Adv. in Math. 4 (1970), 109-126.

[6] D. B. Ray, I. M. Singer, R-torsion and the Laplacian on Riemannian manifolds, Adv. Math 7 (1971), 145-210.

[7] D. B. Ray, I. M. Singer, Analytic torsion, Proceedings of Symposia in Pure Mathematics, vol. 23, p 167, American Mathematical Society 1973.

[8] A. S. Schwarz, I.S. Tyupkin, Quantization of antisymmetric tensors and Ray-Singer Torsion, Nucl. Phys. B 242 (1984) 436.

[9] A. Schwarz, The partition function of a degenerate quadratic functional and Ray-Singer invariants, Lett. Math. Phys, 2 (1978), 247-252.

[10] A. Schwarz, The partition function of a degenerate functional, Commun. Math. Phys. 67 (1979), 1-16.

[11] D. H. Adams, S. Sen, Phase and scaling properties of determinants arising in topological field theories, Phys. Lett. B 353 (1995) no. 4, 495-500, hep-th/9506079.

[12] D. H. Adams, S. Sen, Partition Function of a Quadratic Functional and Semiclassical Approximation for Witten's 3-Manifold Invariant, hep-th/9503095.

[13] D. H. Adams, A note on the Faddeev-Popov determinant and Chern-Simons perturbation theory, Lett.Math.Phys. 42 (1997) 205-214, hep-th/9704159.

[14] D. H. Adams, The semiclassical approximation for the Chern-Simons partition function, Phys.Lett. B417 (1998) 53-60, hep-th/9709147.

[15] D. H. Adams, Perturbative expansion in gauge theories on compact manifolds, hepth/9602078.

[16] C. Nash, D. O' Connor, BRST Quantization and the Product Formula for the RaySinger Torsion, Int.J.Mod.Phys. A10 (1995) 1779-1806, hep-th/9310038.

[17] J. Gegenberg, G. Kunstatter, The Partition Function for Topological Field Theories, Ann. Phys. 231 (1994) 270-289, hep-th/9304016.

[18] M. Blau, G. Thompson, Ann. Phys 205(1991) 130.

[19] C. I. Lazaroiu, R. Roiban and D. Vaman, Graded Chern-Simons field theory and graded topological D-branes, hep-th/0107063.

[20] A. Bondal, M. M. Kapranov, Enhanced triangulated categories, Math. USSR Sbornik, vol 70 (1991) no 1, 93. 
[21] D. E. Diaconescu, M. Douglas, D-branes on Stringy Calabi-Yau Manifolds, hepth/0006224.

[22] M. R. Douglas, D-branes, Categories and N=1 Supersymmetry, hep-th/0011017.

[23] C. I. Lazaroiu, Generalized complexes and string field theory, JHEP 06 (2001) 052.

[24] C. I. Lazaroiu, Unitarity, D-brane dynamics and D-brane categories, hep-th/0102183.

[25] P. S. Aspinwall, A. Lawrence, Derived Categories and Zero-Brane Stability, hepth/0104147.

[26] D. E. Diaconescu, Enhanced D-Brane Categories from String Field Theory, hepth/0104200.

[27] P. S. Aspinwall, M. R. Douglas, D-Brane Stability and Monodromy, hep-th/0110071.

[28] K. Fukaya, Morse homotopy, $A^{\infty}$-category and Floer homologies, in Proceedings of the GARC Workshop on Geometry and Topology, ed. by H. J. Kim, Seoul national University (1994), 1-102; Floer homology, $A^{\infty}$-categories and topological field theory, in Geometry and Physics, Lecture notes in pure and applied mathematics, 184, pp 9-32, Dekker, New York, 1997; Floer homology and Mirror symmetry, I, preprint available at http : //www.kusm.kyoto - u.ac.jp/ fukaya/fukaya.html.

[29] K. Fukaya, Y.-G. Oh, H. Ohta, K. Ono, Lagrangian intersection Floer theory - anomaly and obstruction, preprint available at http : //www.kusm.kyoto u.ac.jp/ fukaya/fukaya.html.

[30] A. Polishchuk, E. Zaslow, Categorical mirror symmetry: the elliptic curve, math.AG/9801119.

[31] M. Kontsevich, Homological algebra of mirror symmetry, Proceedings of the International Congress of Mathematicians, (Zurich, 1994), 120-139, Birkhauser, alggeom/9411018.

[32] E. Witten, Chern-Simons gauge theory as a string theory, The Floer memorial volume, 637-678, Progr. Math., 133, Birkhauser, Basel, 1995, hep-th/9207094.

[33] P. Seidel, Graded Lagrangian submanifolds, Bull. Soc. Math. France 128 (2000), 103149, math.SG/9903049.

[34] J. M. Bismut and J. Lott, Flat vector bundles, direct images and higher analytic torsion, J. Amer. Math Soc 8 (1992) 291.

[35] D. Quillen, Superconnections and the Chern character, Topology, 24, No.1.(1085), 8995. 
[36] C. I. Lazaroiu, String field theory and brane superpotentials, hep-th/0107162.

[37] E. Witten, Topological sigma models, Commun. Math. Phys. 118 (1988),411.

[38] E. Witten, Mirror manifolds and topological field theory, Essays on mirror manifolds, 120-158, Internat. Press, Hong Kong, 1992, hep-th/9112056.

[39] C. I. Lazaroiu, Graded Lagrangians, exotic topological D-branes and enhanced triangulated categories, JHEP 0106 (2001) 064. 